\title{
Úlcera venosa crônica: um relato de caso
}

\author{
Cleide Luciana Toniollo*, Telma Elita Bertolin**
}

\section{Resumo}

Este estudo objetivou relatar uma experiência na terapêutica da úlcera venosa crônica com curativo de poliuretano associado à terapia compressiva. As avaliações e intervenções foram realizadas exclusivamente por uma enfermeira especialista em estomaterapia, utilizando-se de registros fotográficos para o seguimento da evolução da ferida. O estudo foi desenvolvido com autorização do paciente, para uso de imagem a título gratuito. $O$ tratamento durou 60 dias, e durante todo período foi utilizado o mesmo curativo. O paciente apresentava lesão ulcerada há dois anos em membro inferior esquerdo. Na primeira avaliação, a ferida apresentava-se com pouco esfacelo, sinais de gazes no leito da lesão, hiperemia da pele adjacente e edema do membro. $\mathrm{Na}$ terceira troca de Curativo, o paciente já referiu alívio da dor, e a lesão apresentava-se com 100\% de tecido de granulação, edema reduzido e melhora da hiperemia. Aos 60 dias de tratamento com uso de cobertura absorvente associada à terapia compressiva (bota de Unna), toda a extensão da lesão estava em fase final de epitelização. Neste estudo de caso, constatou-se que o curativo de poliuretano e a terapia compressiva inelástica constituíram boas opções para o tratamento da úlcera venosa crônica. A cicatrização ocorreu após 60 dias de tratamento.

Palavras-chave: Úlcera varicosa. Cicatrização. Bandagens.

\section{Introdução}

Estima-se que quase 3\% da população nacional sejam portadores de úlcera venosa, índice que se eleva para $10 \%$ no caso dos diabéticos. Aproximadamente 4 milhões de pessoas são portadoras de lesões crônicas ou têm algum tipo de complicação no processo de cicatrização, o que requer dos profissionais da saúde não só maiores conhecimentos como também preparo para lidar com o problema. (MANDELBAUM; DI SANTIS; MANDELBAUM, 2003).

* Enfermeira especialista em Estomaterapia pela PUCPR e em Administração Hospitalar (Unoesc). Mestra em Envelhecimento Humano pela Universidade de Passo Fundo (UPF). Endereço para correspondência: Rua Uruguai 1471 D, Bairro Saic, CEP: 89802-501 - Chapecó - SC. E-mail: toniollocleide@yahoo.com.br.

** Doutora em Tecnologia Bioquímico-Farmacêutica pela Universidade de São Paulo. Professora titular da Universidade de Passo Fundo no curso de Mestrado em Envelhecimento Humano.

$\hookrightarrow$ doi:10.5335/rbceh.2012.050 
As úlceras venosas podem estar relacionadas a várias doenças. Estas provêm, principalmente, de problemas venosos profundos, em que o aumento crônico da pressão sanguínea intraluminal nos membros inferiores deforma e dilata os vasos, tornando as microvalvas que existem no seu interior ineficientes para o efetivo retorno venoso, ocasionando estase e edema persistentes como resultados do refluxo. (SILVA et al., 2009; FREITAS; JULIÃO, 2006).

A maioria das úlceras de perna obedece a causas vasculares, fundamentalmente à insuficiência venosa de $70 \%$ a $90 \%$ e, de modo menos frequente, à enfermidade oclusiva arterial e diabetes, sendo de 10 a 15\%. (FREITAS; JULIÃO, 2006).

A insuficiência venosa crônica caracteriza-se por alterações físicas, tais como edema, hiperpigmentação, eczema, erisipela, lipodermoesclerose, que se manifestam na pele e no tecido subcutâneo, principalmente nos membros inferiores, devido à hipertensão venosa de longa duração. A pressão elevada no interior do vaso afeta a microcirculação, aumentando a permeabilidade dos vasos, o que permite a liberação de substâncias do seu interior para a pele e resulta nessas manifestações, cujo estágio mais avançado e grave é a formação de úlcera. (ABBADE; LASTÓRIA, 2006).

$\mathrm{O}$ diagnóstico definitivo da úlcera venosa pode ser feito por meio de alguns exames invasivos ou não invasivos; porém, na maioria das vezes, o exame físico e a história do paciente são suficientes para concluir o diagnóstico clínico e orientar o tratamento. Os traumatis- mos podem ser fatores desencadeantes, por isso é importante questionar o paciente quanto à história de fraturas de membros inferiores tratadas com gesso. Geralmente, as úlceras estão localizadas nas extremidades, região dos maléolos internos e com presença de edema no tornozelo, principalmente ao final do dia. Os pacientes acometidos por essas lesões, frequentemente, queixam-se de dor, relatando melhora com a elevação dos membros inferiores. (ABBADE; LASTÓRIA, 2006).

O tratamento das úlceras venosas pode ser cirúrgico ou clínico e dá-se no controle da hipertensão deambulatória. Porém, devido à dificuldade de acesso ao procedimento cirúrgico, os pacientes passam longos períodos realizando o tratamento clínico, que inclui, necessariamente, a terapia compressiva para facilitar o retorno venoso. Uma das formas de tratamento compressivo inelástico é a bota de Unna. (SILVIA; FIGUEIREDO; MEIRELES, 2007).

Aproximadamente $75 \%$ das úlceras de perna resultam de insuficiência venosa crônica, sendo $20 \%$ causadas por insuficiência arterial e 5\% provocadas por outros fatores. (FREITAS; JULIÃO, 2006). Essa síndrome, que envolve a destruição de estruturas cutâneas, tais como epiderme e derme, podendo afetar, também, tecidos mais profundos, manifesta-se no terço inferior dos membros inferiores. (MALAGUTTI; KAKIHARA, 2010). Essa inadequação do funcionamento do sistema venoso é comum na população idosa, sendo a frequência superior a $4 \%$ entre os idosos acima de 65 anos. (SANTOS, 2000). 
Existem, atualmente, várias opções de tratamento para esse tipo de enfermidade, estando já bem definidas e estabelecidas no contexto da medicina as indicações terapêuticas e profiláticas, como as meias elásticas, ataduras e compressões pneumáticas. O International Compression Club referendou essas evidências em reunião realizada em Paris, em novembro de 2007. (PARTSCH; FLOUR; SMITH, 2008). A seguir, tem-se as indicações e o uso pós-procedimentos da compressão de acordo com os graus de evidência 1A e $1 \mathrm{~B}$ recomendados. (GUYATT; GUTTERMAN; BAUMANN, 2006).

O grau de evidência para uso da meia com 20 mmHg de compressão é:

- 1B na prevenção do edema ocupacional;

- 1B em casos iniciais CEAP (clínica, etiológica, anatômica e fisiopatológica) (C0 s e C1 s);

- 1 A na prevenção do tromboembolismo venoso.

O grau de evidência para uso da meia com 20-30 mmHg de compressão é:

- 1B em pós-escleroterapia em microvarizes;

- 1B na prevenção dos sintomas de insuficiência venosa durante a gestação;

- $1 B$ na prevenção do edema venoso em pacientes CEAP 3.

O grau de evidência para uso da meia com 30-40 mmHg de compressão é:

- 1B no tratamento de trombose venosa profunda;

- 1 A na prevenção da síndrome pós-flebítica;

- 1B no tratamento do paciente CEAP 4;
- 1B no tratamento da úlcera venosa aberta;

- 1A na prevenção de recidiva da úlcera (CEAP 5).

O grau de evidência para uso de ataduras elásticas e inelásticas é:

- 1Ana cicatrização das úlceras venosas com bota de Unna ou curativo multicamadas.

As úlceras venosas como doença crônica e seu impacto na economia possuem relevância pelo fato de sua prevalência e incidência serem universais e por terem características de recorrência. A significativa morbidade que essa patologia acarreta constitui importante causa de desconforto e incapacidade, impactando negativamente a qualidade de vida dos pacientes, inclusive na vida social e no trabalho. (BORGES, 2005).

Pode-se afirmar que as úlceras venosas "causam danos aos pacientes porque afetam seu estilo de vida devido à dor, depressão, perda da autoestima, isolamento social, inabilidade para o trabalho e, frequentemente, hospitalizações ou visitas clínicas ambulatoriais". (ARON, 2005). Para muitos pacientes, a úlcera venosa significa isolamento social e efeito emocional negativo, por desencadear no indivíduo constrangimento, tristeza, raiva, autoimagem negativa. (PARTSCH; FLOUR; SMITH, 2008).

Em contrapartida, existem pesquisas que demonstram outra realidade. Em estudo realizado com 89 pessoas, no período de setembro de 2000 a março de 2001, obteve-se resultado diferente em relação à qualidade de vida de portadores de úlcera venosa crônica. Dos participantes da pesquisa, $37(41,7 \%)$ e 
$35(39,31 \%)$ apresentaram, respectivamente, qualidade de vida considerada como "muito boa" e "boa". As divergências apresentadas resultam do fato de a qualidade de vida ser marcada pela subjetividade e multidimensionalidade; portanto, sua avaliação dependerá dos valores e das concepções do indivíduo, bem como dos contextos diferenciados nos quais as pessoas estão inseridas. (BORGES, 2005).

Esse aspecto pode ser observado na convivência com esses pacientes no serviço de estomaterapia, conhecendo a história e os casos de cada paciente. Para os profissionais da enfermagem, a assistência a esses pacientes é um grande desafio, exigindo estratégias de melhoria para saúde e qualidade de vida, por meio de tratamento individualizado e integrativo a partir da colaboração e adesão do paciente. Portanto, contribuir com a prática clínica no manejo das úlceras venosas, por meio do relato de experiência de um caso, é o que justifica a realização deste estudo, cujo objetivo consiste em apresentar um estudo de caso referente ao tratamento de paciente com úlcera venosa crônica, utilizando cobertura de poliuretano impregnada com prata associada a terapia compressiva inelástica (bota de Unna).

\section{Métodos}

Trata-se de um estudo de caso com abordagem avaliativa, realizado em um hospital particular na cidade de Chapecó, região Oeste de Santa Catarina, tendo duração de 60 dias, entre os meses de novembro de 2008 e janeiro de 2009 . A amostra foi constituída de uma úlcera venosa de um paciente submetido ao tratamento com cobertura de poliuretano após limpeza das lesões com solução de soro fisiológico a $0,9 \%$. A variável analisada foi a área lesada, considerando o seu aumento ou sua redução no decorrer do período em avaliação em porcentagem.

A coleta de dados foi efetuada por meio de registro fotográfico em máquina digital, tendo as fotos sido realizadas antes do início e no decorrer do tratamento. As observações foram anotadas em prontuário, especificamente em impressos usados para a Sistematização da Assistência de Enfermagem, antes do início do seguimento do paciente e a cada troca de curativo, objetivando analisar a evolução da ferida.

Solicitou-se autorização ao paciente, por escrito, para uso de imagem a título gratuito, precedida de explicação verbal sobre os objetivos e métodos do estudo, conforme Resolução 196/96 do Conselho Nacional de Saúde, que dispõe sobre a ética em pesquisa com seres humanos. A realização do trabalho foi aprovada pelo serviço onde os dados foram coletados ${ }^{1}$.

\section{Caso clínico}

Paciente com 66 anos, sexo masculino, agricultor, natural de Chapecó, residente na Linha Alta da Serra, interior do município, com leucemia diagnosticada há quatro anos e dois meses, hipertenso há três anos, em uso de medicação para ambas as doenças, recentemente diagnosticada neoplasia de próstata, apresenta antecedentes de tabagismo por 25 anos, tendo parado há 22 anos, nega etilismo. 
Na primeira avaliação, o paciente relatou que apresentava a úlcera há dois anos, de origem venosa, localizada em membro inferior esquerdo (MIE), tendo sido submetido a vários tratamentos tópicos e medicações analgésicas anteriormente, sem obter melhora da lesão. Apresentava-se normotenso, em bom estado geral.

A úlcera apresentava-se com $6 \mathrm{~cm}$ de diâmetro, bordas planas e irregulares, leito com presença de tecido de granulação e poucos esfacelos, exsudato serosanguinolento em grande quantidade, no terço inferior do MID, região maleolar, presença de edema, pele adjacente hiperemiada e queixa de dor intensa (Figura 1).

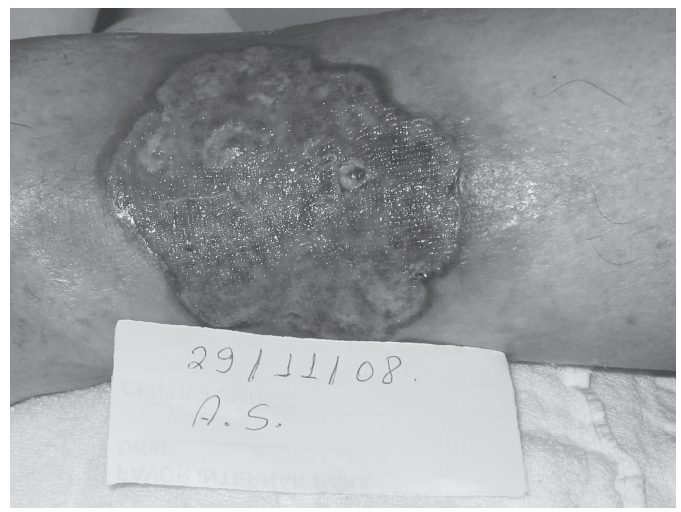

Figura 1 - Úlcera venosa na primeira avaliação, presença de esfacelo no leito, hiperemia e edema no membro.

Fonte: Arquivo pessoal do autor (2008).

Após a avaliação física e detecção de sinais clínicos específicos da insuficiência venosa crônica, verificou-se o índice tornozelo braquial (ITB) com resultado que confirmou a origem venosa, facilitando assim a escolha do tratamento adequado.
Inicialmente, orientou-se ao paciente repouso, com elevação dos membros inferiores por 40 minutos com o objetivo de reduzir o edema, em seguida foi realizado curativo respeitando os seguintes passos: limpeza da ferida com SF 0,9\% morno; aplicação da cobertura primária com espuma de poliuretano e aplicação de bandagem inelástica confeccionada com pasta de Unna composta por glicerina, gelatina, cosmoguard $0,1 \mathrm{ml}$ e óxido de zinco, esse procedimento foi repetido até o final do tratamento.

A terapia compressiva auxilia no processo de cicatrização das úlceras venosas sendo que pode representar a melhor opção quando se trata de pacientes sem doença arterial. (ARON, 2005; SILVIA; FIGUEIREDO; MEIRELES, 2007).

No que diz respeito à limpeza da lesão, esta pode ser feita com soro fisiológico a $0,9 \%$, morno em jato para garantir limpeza eficaz e minimizar os riscos de trauma adicional na lesão. (POLETTI, 2000).

Já no que concerne à cobertura para a úlcera venosa, esta deve absorver o exsudato do leito da lesão, manter ambiente local úmido, ser de fácil aplicação e remoção a fim de evitar traumas durante a troca, minimizar a dor da ferida, ser hipoalergênica, ser impermeável a patógenos, ser estéril e livre de contaminantes, bem como prover isolamento térmico (CARMO et al., 2007), sendo a espuma de poliuretano a cobertura que preencheu essas características.

Quanto à forma de terapia compressiva inelástica apresenta-se a Bota de Unna. Para que se utilize esse tipo de compressão é necessário que a ferida 
esteja instalada, pois após sua cura o paciente deve usar a meia de compressão com o intuito de evitar recidiva. (LOPEZ; ARAVITES; LOPES, 2005).

Sendo assim, os curativos foram realizados pelo período de duas semanas, com intervalo de quatro dias entre as trocas, após o décimo oitavo dia, ou seja, na quarta troca de curativo, foi evidenciada a redução do exsudato, ausência de hiperemia e a presença de tecido de granulação em toda a área da ferida (100\%), além disso, houve o alívio completo da dor permitindo a suspensão das medicações analgésicas, sendo que anteriormente estas eram empregadas diariamente (Figura 2).

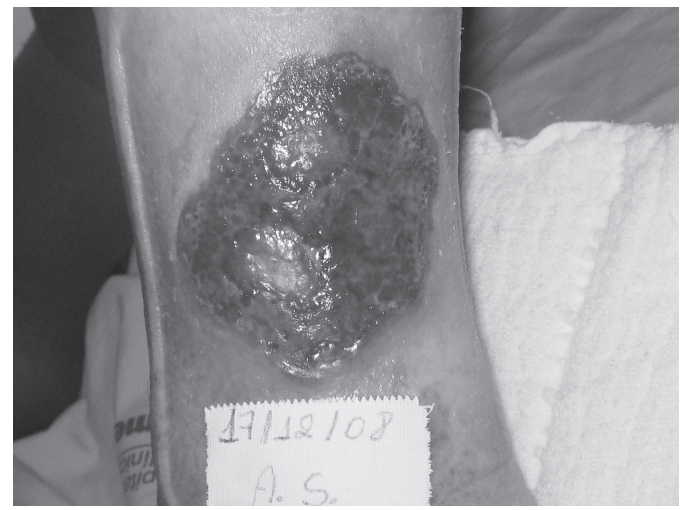

Figura 2 - Úlcera venosa recoberta com tecido de granulação, redução da hiperemia e do edema (após 18 dias de tratamento tópico).

Fonte: Arquivo pessoal das autoras (2008).

Após 18 dias, as trocas de curativo passaram a ser realizadas com intervalos de sete dias.

Passados 30 dias de seguimento, $60 \%$ da ferida estava epitelizada, havia pequena quantidade de exsudato, ausência de edema, inexistência de dor e presença de $100 \%$ de tecido viável (Figura 3).

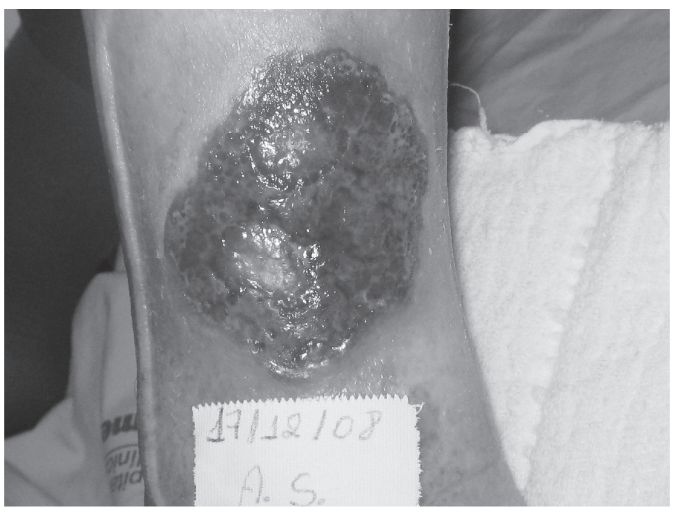

Figura 3 - Epitelização da lesão, após 30 dias de seguimento.

Fonte: Arquivo pessoal das autoras (2008).

Já aos 60 dias de tratamento, toda a extensão da lesão estava em fase final de epitelização, não havia presença de exsudato, nem de edema e dor (Figura 4).

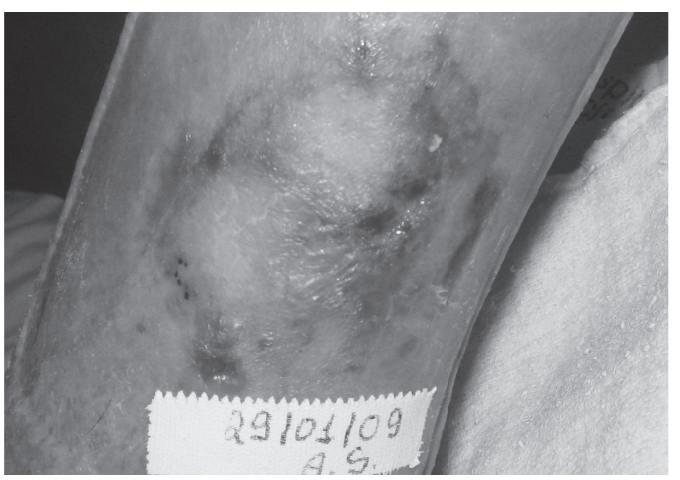

Figura 4 - Ferida em fase final de epitelização e cicatrização, após 60 dias de tratamento.

Fonte: Arquivo pessoal das autoras (2008).

\section{Discussão}

Os pacientes com úlcera venosa têm várias opções de coberturas e limpeza, dentre as quais, destacam-se: hidroco- 
loides, alginatos, hidrogel, espuma de poliuretano com prata e carvão ativado. (SANTOS, 2000; PARTSCH; FLOUR; SMITH, 2008). Já para a limpeza, podem ser utilizadas água, solução fisiológica ou solução de papaína, desde que de qualidade e livres de contaminantes. (LOPEZ; ARAVITES; LOPES, 2005; YAMADA, 1999; ARON, 2005).

No estudo realizado, para limpeza da ferida foi utilizada solução fisiológica ( $\mathrm{NaCl} 0,9 \%)$ morna $\left(37^{\circ} \mathrm{C}\right)$ aplicada em jato, utilizando seringa de $20 \mathrm{ml}$ com agulha 40 x 12 durante os 60 dias de tratamento.

A técnica empregada no estudo seguiu a recomendação de diferentes autores (BORGES, 2005; ROGENSKI; BAPTISTA; SOFIA, 1998; LOPEZ; ARAVITES; LOPES, 2005; AHCPR, 1992), que informam que as soluções utilizadas para limpeza da ferida devem ser preferencialmente aquecidas para evitar a redução da temperatura no leito da lesão. Uma temperatura constante de 37 graus estimula a mitose durante a granulação e epitelização.

Aliado às coberturas e técnicas de limpeza, outro recurso empregado foi a terapia compressiva, especificamente a bota de Unna, a qual representa uma forma de terapia inelástica. A troca dessa bandagem deve ser realizada uma ou duas vezes por semana, dependendo do volume do exsudato. (PARTSCH; FLOUR; SMITH, 2008).

Em um estudo realizado por Partsch, Flour e Smith (2008), a melhor taxa de cicatrização foi obtida com a utilização de bandagem de alta compressão (40 mm $\mathrm{Hg}$ ), sendo essa a primeira escolha para o tratamento de úlceras venosas não complicadas, com uso indicado pelo tempo mínimo de uma semana.

\section{Conclusão}

A aplicação dessa técnica demonstrou ser eficaz, pois propiciou melhora significativa da úlcera em todas as suas características; ou seja, como resultado, obteve-se redução do tamanho da lesão, controle do exsudato, alívio da dor, redução do edema e melhora da autoestima referida pelo paciente (embora não tenha sido avaliada por meio de instrumento específico). Cabe salientar que a escolha adequada da terapia tópica é de fundamental importância para facilitar e acelerar o processo cicatricial, minimizando o desconforto pela presença da ferida e, certamente, impactando positivamente na qualidade de vida do paciente.

\section{Chronic venous ulcer: a case report}

\section{Abstract}

This study describes the author's experience in the treatment of chronic venous ulcers with compression therapy and wound dressing made of polyurethane. Assessments and interventions were carried out exclusively by a nurse specialist Stomatherapy, using photographic records to follow the evolution of the wound. The study was performed with the patient's authorization to use picture for free. The treatment lasted for sixty days and throughout the same period was a used dressing. The patient had an ulcer two years ago in the left leg. In the first, the wound presented with little slough, signs of gauze in the wound bed, skin hyperemia and edema of the adjacent member. In the third dressing change, the patient already reported relief of pain and the lesion was $100 \%$ granulation tissue, reduced edema and improvement in hyperemic. At sixty days of treatment with the use of absorbent covering associated with 
compression therapy (Unna boots), full extent of the lesion was in the final stages of healing. In this case study, it was found that the polyurethane dressing and inelastic compression therapy were good options for the treatment of chronic venous ulcers. Healing occurred after sixty days of treatment.

Keywords: Varicose ulcers. Healing. Bandages.

\section{Notas}

1 No período em que o estudo foi realizado, não havia comitê de ética na instituição, e o projeto não foi submetido a nenhum outro comitê. Portanto, o sigilo das informações é de inteira responsabilidade das autoras.

\section{Referências}

ABBADE, L. P. F; LASTÓRIA, S. Abordagem de pacientes com úlcera da perna de etiologia venosa. Anais Brasileiros de Dermatologia, São Paulo, v. 6, n. 81, p. 509-522, dez. 2006. Disponível em: <http://www.scielo.br/ scielo.php?script=sci_arttext\&pid=S0365$-05962006000600002 \& l n g=e m>$. Acesso em: 26 jun. 2010.

AGENCY FOR HEALTH CARE POLICY AND RESEARCH (AHCPR). Clinical practice guideline n. 3: pressure ulcers in adults prediction and prevention - U.S. Department of Health and Human Services. Publication n. 92-0047, May 1992.

ARON, S. O uso das meias de compressão no tratamento de úlceras venosas. Estima, São Paulo, v. 3, n. 4, p. 43-45, 2005.

BORGES, E. L. Tratamento tópico de úlcera venosa: proposta de uma diretriz baseada em evidências. 2005. 305 f. Tese (Doutorado em Enfermagem) - Escola de Enfermagem/USP, Ribeirão Preto, 2005.
CARMO, S. S. et al. Atualidades na assistência de enfermagem a portadores de úlcera venosa. Revista Eletrônica de Enfermagem, Goiânia, v. 9, n. 2, p. 506-551, 2007. Disponível em: <http://www.fen.ufg.br/revista/v9/ n2/v9n2a17.htm>. Acesso em: 26 jun. 2010.

FREITAS, M. A. S.; JULIÃO, M. C. C. Transversal escharotomies: a new surgical technique adjuvant in the treatment of chronic ulcers with non-arterial etiology in the lower limbs. Acta Cirúrgica Brasileira, São Paulo, v. 21, supl. 4, 2006. Disponível em: <http://www.scielo. br/scielo.php?script=sci_arttext\&pid=S0102$-86502006001000018 \& \operatorname{lng}=\mathrm{em}>$. Acesso em: 26 jun. 2010.

GUYATT, G.; GUTTERMAN, D.; BAUMANN, M. H. Grading strength of recommendations and quality of evidence in clinical guidelines: report from an American College of Chest Physicians Task Force. Chest., v. 129, n. 1, p. 174-181, 2006.

LOPEZ, A. R.; ARAVITES, L. B.; LOPES, M. R. Úlcera venosa. Acta Médica, Porto Alegre, n. 26, p. 331-341, 2005.

MALAGUTTI, W.; KAKIHARA, C. Curativos, estomias e dermatologia: uma abordagem multiprofissional. São Paulo: Martinari, 2010.

MANDELBAUM, S. H.; DI SANTIS, E. P.; MANDELBAUM, M. H. S. Cicatrização: conceitos atuais e recursos auxiliares - Parte I. Anais Brasileiros de Dermatologia, Rio de Janeiro, v. 78, n. 4, p. 393-410, ago. 2003.

PARTSCH, H.; FLOUR, M.; SMITH, P. C. International compression club. Indications for compression therapy in venous and lymphatic disease consensus based on experimental data and scientific evidence. International Angiology, v. 27, n. 3, p. 193219, 2008.

POLETTI, N. A. A. O cuidado de enfermagem a pacientes com feridas crônicas: a busca de evidências para a prática. 2000. $237 \mathrm{f}$. Dissertação (Mestrado em Enfermagem Fundamental) - Escola de Enfermagem/USP, Ribeirão Preto, 2000. 
ROGENSKI, N. M. B.; BAPTISTA, C. M. C.; SOFIA, M. H. O uso da papaína a $2 \%$ nas lesões provocadas pela Síndrome de Fournier: a propósito de 14 casos. Revista Paulista de Enfermagem, São Paulo, v. 17, n. 1, p. 39-45, 1998.

SANTOS, V. L. C. G. Avanços tecnológicos no tratamento de feridas e algumas aplicações em domicílio. In: DUARTE, Y. A. O.; DIOGO, M. J. D. Atendimento domiciliar: um enfoque gerontológico. São Paulo: Atheneu, 2000. p. 265-306.

SILVA, F. A. A. da et al. Enfermagem em estomaterapia: cuidados clínicos ao portador de úlcera venosa. Revista Brasileira de Enfermagem, Brasília, v. 62, n. 6, p. 889-893, dez. 2009. Disponível em: <http://www.scielo.br/ scielo.php?script=sci_arttext\&pid=S0034716 $72009000600014 \& l n g=e n \& n r m=i s o>$. Acesso em: 21 abr. 2012.

SILVIA, R. C. L.; FIGUEIREDO, N. M. A.; MEIRELES, I. B. Feridas: fundamentos e atualizações em enfermagem. São Caetano do Sul: Yendis, 2007.

YAMADA, B. F. A. Terapia tópica de feridas: limpeza e desbridamento. Revista da Escola de Enfermagem, São Paulo, v. 33, p. 133-140, 1999. 